\title{
REVISIÓN: LESIONES DE RODILLA EN CICLISMO DE CARRETERA
}

\section{REVIEW: KNEE INJURIES IN CYCLING ROAD}

\author{
Rodríguez Rodríguez, Fernando
}

\begin{abstract}
Laboratorio de Motricidad Humana, Escuela de Educación Física, Laboratorio de Antropología Física y Anatomía Humana, Instituto de Biología, Pontificia Universidad Católica de Valparaíso, Chile
\end{abstract}

RODRIGUEZ, R.F. Revisión: Lesiones de rodilla en ciclismo de carretera. Rev. Motr. Hum., 10(2): 54-60, 2009.

\begin{abstract}
RESUMEN
El ciclismo es, ciertamente, una actividad deportiva de bajo impacto, pero que realizada bajo condiciones inadecuadas como una bicicleta muy pesada, usar el asiento bajo, desalinear los pedales, falta de una condición física adecuada o una morfología incompatible; es capaz de provocar lesiones por sobreuso, que existen en muñecas, brazos, hombros, espalda, rodillas y tobillo. La más imposibilitante es la de rodilla, siendo las más comunes son la Tendinopatía del Tendón Patelar (TTP) y el Síndrome de la Compresión Patelo-Femoral (SCPF). Para ambas lesiones en etapas iniciales funciona el tratamiento convencional, pero lo importante es la prevención de dichas lesiones, teniendo el conocimiento adecuado de las posiciones y cargas de entrenamiento adecuadas para cada individuo.
\end{abstract}

Palabras Clave: Ciclismo, Tendinopatías, Compresión patelo-femoral.

\section{INTRODUCCIÓN}

El estudio del ciclismo está reforzado por varias disciplinas del conocimiento, como la mecánica y física de fluidos hechos en los túneles de viento que inicialmente se crearon con objetivo de mejorar la aeronáutica (1,2); los estudios fisiológicos y de medicina deportiva; los estudios de mecánica muscular (3); y la biomecánica y cinemática del pedaleo óptimo y eficaz $(4,5)$.

La interacción entre ciclista y bicicleta nos permite distinguir tres fuerzas externas de resistencia al desplazamiento: las producidas por el aire, las de fricción entre el neumático y el piso, y las que ejerce la gravedad cuando se desplaza en una pendiente. En menor medida se debe vencer la resistencia entre la cadena los engranajes, la cual no llega al 5\% del total de las fuerzas de resistencia $(6,7)$.

Se puede, además, considerar como fuerza propulsiva externa a la acción de la gravedad cuando el sistema se desplaza descendiendo una pendiente, el resto de las fuerzas propulsivas se consideran interactivas dentro del sistema, como las ejercidas sobre el sillín, manillar y especialmente sobre los pedales, teniendo en cuenta que la génesis de dichas fuerzas propulsivas reside en la contracción muscular y los momentos de fuerza que se ejercen sobre las articulaciones.
Al margen de la energía consumida en los engranajes y el efecto producido por la gravedad al ir en una pendiente, las dos grandes fuerzas que se oponen al desplazamiento son las producidas por el contacto de la rueda con el suelo y las ejercidas por el aire (aerodinámicos).

Para comprender la importancia que tienen las resistencias producidas por el aire en ciclismo, se pueden exponer algunos datos que nos revelan el costo energético que supone desplazar algunos cientos de kilos de aire por minuto. Mientras que el récord de velocidad en ciclismo deportivo sobre 200 metros (m.) es de 71,29 kilómetros por hora $(\mathrm{km} / \mathrm{h})$, establecido por Adamashvili en 1990; el récord conseguido por un vehículo aerodinámico fuselado es de 105, $39 \mathrm{~km} / \mathrm{h}$, establecido por Markham en 1986. Un año antes, el norteamericano Howard consiguió alcanzar los 245,08 km/h corriendo detrás de un protector de aire. Otro dato comparado que permite significar la importancia que adquieren las resistencias aerodinámicas es el récord de la hora, mientras que el record oficial lo consiguió en noviembre de 1994 el suizo Rominger en 55,291 kilómetros $(\mathrm{km}$.$) , el récord de la hora en pista$ cubierta, tras moto, lo consiguió en 1987 el ruso Romanov en 91,131 km., algo impensable de cubrir en situaciones normales. Los datos expuestos reflejan que la mayor fuerza opuesta al desplazamiento del 
ciclista es la aerodinámica, esta supone más del $80 \%$ de todas las fuerzas de resistencia cuando sólo se desplaza a $30 \mathrm{~km} / \mathrm{h}$ y muy superiores cuando se incrementa la velocidad.

Se puede afirmar que la fuerza aerodinámica más importante que se opone al desplazamiento en ciclismo es la oponente de arrastre, la cual puede considerarse compuesta de un arrastre viscoso o superficial producido por la fricción laminar del aire contra la bicicleta y el ciclista, más un arrastre de forma producido por el déficit de momento de la estela al existir separación de las líneas de corriente.

Cuando un ciclista se desplaza a cierta velocidad, las capas de aire más próximas se desplazarán con él, produciendo un cambio gradual de velocidad entre capas y, consecuentemente, un deslizamiento que se traduce en el incremento del arrastre viscoso y superficial.

Estas fuerzas mecánicas que vencer necesitan de un desarrollo energético importante, conseguido por un entrenamiento aeróbico riguroso, que se expresa por medio de fuerza y resistencia muscular que son absorbidos por los componentes blandos como músculos, tendones y ligamentos; provocando una sobrecarga que no todos los ciclistas son capaces de soportar y que desarrollan inflamaciones dolorosas que terminan en lesiones crónicas con dolor agudo.El ciclismo es considerado un deporte de bajo impacto, pues en otros deportes la articulación de la rodilla es la que recibe la carga y fuerza aplicada por el sistema muscular. Una revisión a las complicaciones en la rodilla del ciclista se realizará a continuación.

\section{LESIONES POR SOBREUSO}

Durante la práctica del ciclismo un deportista bien entrenado realiza entre 95 a 105 pedaladas por minuto por cada extremidad, considerando que se entrena normalmente por 3 horas, los ciclistas ejecutan entre 17.000 y 19.000 pedaladas y en las grandes vueltas europeas estas cifras superan largamente las 20.000 pedaladas.

Existen, además, condicionantes morfológicas y fisiológicas que aumentan el riesgo de padecer estas lesiones, como el nivel de competición en el que participe el ciclista, el tipo de calzado utilizado, la dominancia de las extremidades, la condición física, el peso corporal y la flexibilidad, entre otras (8).

La gran cantidad de rozamiento y carga duradera, conlleva a dos condiciones generalmente dolorosas típicas de los ciclistas, la Tendinopatía del Tendón Patelar (TTP) y la Compresión Patelo-Femoral (CPF).

\section{TENDINOPATIA DEL TENDÓN PATELAR}

La TTP es comúnmente conocida como la rodilla del saltador, ya que es frecuente en deportes que involucran saltos como el básquetbol o el voleibol, pero también se puede presentar en otros deportes como el fútbol o, en este caso, en el ciclismo.

Es raro que el dolor se presente a lo largo de todo el tendón patelar, generalmente los pacientes tienen dolor en la parte inferior de la patela y cercana a su vértice en donde comienza el tendón patelar en la tibia.

Hay ciertos factores que se asocian a ésta enfermedad, como la "Patela Alta" (9). La patela se encuentra en una posición más alta que lo normal, hay una falta de desarrollo de la porción interna del cuádriceps (Vasto medial).

La TTP es causada principalmente por ejecuciones repetidas de un movimiento, en este caso de la extensión de la rodilla, que se caracteriza por la degeneración del tendón que provoca un proceso inflamatorio que demuestra una degeneración del colágeno y aumento de mucosas (10).

Según Blazima (11), las TTP se clasifica en 4 grados: Grado I, dolor leve después de finalizado el ejercicio; Grado II, dolor antes del ejercicio, mejora durante el ejercicio y empeora más al final del ejercicio; Grado III, dolor durante y después del ejercicio perjudicando el rendimiento físico y; Grado IV, ruptura parcial o total del tendón.

Una vez adquirida la lesión se ve favorecida por cambios bruscos en la tensión del tendón como en el cambio de terreno llano a terreno en montaña, el viento en contra o la ejecución de sprints finales. Otro factor que aumenta los síntomas es el cambio de temperaturas (calor -frío). 
Inicialmente, y cuando los síntomas son de reciente aparición, evitar la causa del micro trauma, o el sobreesfuerzo al que generalmente es sometido el tendón en los entrenamientos, es suficiente para revertir el proceso. En este estadio es habitual que el deportista no quiera someterse a tratamiento o a la limitación de su actividad, ya que el dolor es mínimo y no influye sobre el rendimiento deportivo. Es en estos atletas y en este momento en que el poder evaluar la etiología y la orientación sobre la forma y cantidad de entrenamiento y uso es realmente efectivo y puede evitar que la patología progrese.

La Tendinitis Patelar Crónica puede ser un problema muy difícil de tratar. El tratamiento quirúrgico solo se reserva para los casos recalcitrantes en que el tratamiento conservador ha fracasado. En éstos casos se realiza una técnica quirúrgica mixta, se hace una revisión artroscópica de la rodilla y posteriormente se hace una incisión sobre el tendón patelar y se limpia. Los resultados para los casos crónicos rebeldes al tratamiento conservador son buenos sin llegar a ser excelentes.

Otra alternativa de tratamiento es la inmovilización con yeso, que no es totalmente recomendada ya que la inactividad en un tejido elástico como el tendón, predispone a los cambios histológicos en el tejido conectivo y la elastina del tendón. Probablemente, el reposo absoluto disminuya la resistencia del tendón y sus inserciones óseas, inhibiendo los procesos de cicatrización.

Los antiinflamatorios no esteroides (AINEs) colaboran con el proceso desinflamante utilizándolos aproximadamente por dos semanas. El resultado de esta medicación es una mejor respuesta cuando el proceso se encuentra en su estadio agudo y no en el crónico.

El tratamiento de la inflamación con corticoides ha sido utilizado con frecuencia para curar esta afección. Todos los recientes estudios coinciden en que el corticoide local actúa en un proceso de degeneración del tejido, por lo que su utilización no es recomendable, aún en estadios iniciales. Los más utilizados son: ácido acetilsalicílico, ibuprofeno, naproxeno, piroxicam, fenoprofeno, tolmetina, fenilbutazona (12).
Tradicionalmente se ha usado el reposo, hielo, y la fisioterapia (fortalecimiento del vasto medial, estiramiento de isquiotibiales y cuádriceps) con resultados aceptables.

El Dr. Bill Stanish de Halifax Nueva Escocia, quien tiene una amplia experiencia en el tema, ha encontrado que sometiendo a los pacientes con Tendinitis Patelar a un programa de EJERCICIOS EXCÉNTRICOS DEL CUÁDRICEPS, mejoran notablemente. También mencionó que en éstos pacientes hay una disminución de la fuerza muscular de los dorsiflexores del tobillo, por lo que también hay que fortalecer éstos músculos. El Dr. Stanish usa, además de todo lo anterior, el masaje profundo con un cubo de hielo (13).

La fisioterapia (FT) con el ultrasonido, láser o magnetoterapia colaboran aumentando la circulación local, estimulando el proceso desinflamatorio. $\mathrm{Su}$ acción es eficaz también por lo superficial de la lesión y la efectiva acción de la FT sobre la zona.

Los ejercicios excéntricos y el fortalecimiento controlado de los músculos extensores se indican bajo supervisión del terapeuta. El evaluar desbalances musculares colabora posibilitando con el entrenamiento un equilibrio muscular para evitar la recidiva.

La tendencia actual busca una mayor extensión de la rodilla en el pedaleo, superando los 160 grados $\left({ }^{\circ}\right)$ de extensión. Es importante evitar en el esfuerzo el movimiento de retroceso en el sillín, para que la palanca en extensión sea más efectiva. El pedaleo debe ser "redondo", es decir, la fuerza se debe aplicar tanto al extender la rodilla como al flexionarla, a pesar de que esto no aporta una mejora en la eficiencia el pedaleo, evita la lesión al descargar la tensión de músculo cuádriceps y del tendón correspondiente, repartiendo la energía en los músculos posteriores del muslo (isquiotibiales) y flexores de pierna (gastrocnemio, poplíteo y plantar). Este error en la aplicación de la fuerza en sólo los músculos extensores de la rodilla es común en ciclistas amateur, que se están iniciando en el deporte o que realizan ciclismo recreativo. 


\section{SINDROME DE COMPRESIÓN PATELOFEMORAL (SCPF)}

La SCPF consiste en una gran presión de la patela contra el fémur debido a la contracción del cuádriceps y la resistencia que opone el tendón patelar y que representa un tercio de las lesiones deportivas de rodilla $(14,15)$.

Cada superficie articular del fémur y de la patela posee tejido de colágeno tipo II, conocido como cartílago articular, que tiene la doble función de amortiguar e impedir el choque óseo entre ambas superficies articulares, así como también favorecer con su suave capa externa el deslizamiento adecuado de la patela sobre el fémur. Una de las principales causas del SCPF es el exceso en la flexión de la rodilla, cuando el sillín está muy bajo, finalizando en una lesión crónica conocida como condropatía de patela. Las presiones femoropatelares son muy intensas, del orden de 256 kilogramos (kg.) en los $40^{\circ}$ de flexión a los $420 \mathrm{~kg}$. a $55^{\circ}$ de flexión para un ciclista de $60 \mathrm{~kg}$. Estas presiones de sobrecarga, unidas a la contracción del cuádriceps y a la resistencia del tendón patelar aplastan la rótula con fuerza sobre la tróclea femoral (16).

La consecuencia más usual del no tratamiento del SCPF es la condromalasia, que es el daño y pérdida de las funciones del cartílago (17), y que pueden progresar una pérdida total del cartílago provocando una ruptura ósea denominada osteoartrosis.

En presencia de este síndrome, se observa dolor a lo largo de la carilla lateral de la patela o del cóndilo femoral, presentando derrame cuando el cartílago está dañado. Históricamente, el inicio del síndrome patelofemoral ha sido atribuido solamente a un aumento del ángulo Q que está formado por una línea que va desde la tuberosidad de la tibia al centro de la patela y por otra línea que va desde la espina ilíaca anterosuperior al mismo centro de la patela. Pero la función patelofemoral se fundamenta en diversas variables individuales, cada cual con su propia influencia en el mecanismo extensor (18).

Es necesario mencionar que el ángulo $\mathrm{Q}$ es mayor en mujeres que en varones, por lo tanto el riesgo de sufrir SCPF aumenta. Esto, normalmente a causa de una inclinación mayor del fémur provocando, a su vez, una pelvis más ancha.

Otro factor importante en la generación del SCPF es la forma que posee la patela y las caras articulares del fémur, que pueden ser perfectamente convergentes o tener superficies opuestas. Se distinguen tres tipos de patelas: el tipo I presenta carillas lateral y medial similares; el tipo II tiene una carilla medial más pequeña, plana o ligeramente convexa; y el tipo III tiene una carilla medial muy reducida y convexa.

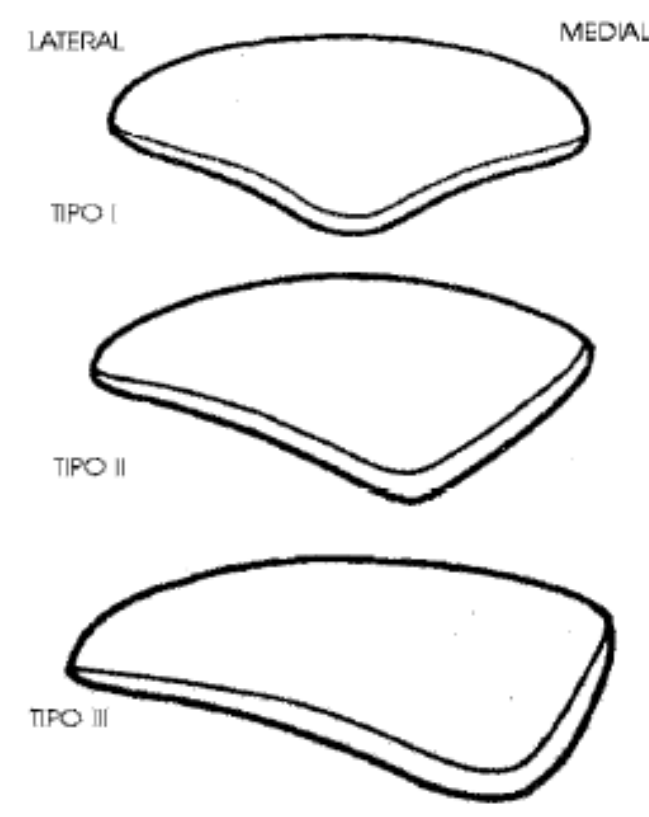

Figura 1: Tipología y morfología patelar según Wiberg (19)

Como las caras articulares del fémur son convexas, las patelas tipo II y tipo III tienen menor coaptación articular, por lo que provocan mayor incidencia de lesiones.

Después de realizar la anamnesis, donde deberá constar: el inicio de la lesión, la localización del dolor, las actividades que exacerban el dolor, nivel de actividad, lesiones y cirugías previas y el mecanismo de la lesión; se debe proceder con el examen físico (18). 
Según Starkey \& Ryan (18), en la inspección se debe evaluar el alineamiento de la patela, el alineamiento defectuoso de la rótula, el tamaño del tendón patelar, postura de la rodilla, ángulo $\mathrm{Q}$, diferencia entre las medidas de las piernas y posicionamiento de los pies. Con relación a la palpación, se debe evaluar la tuberosidad tibial, la forma de la patela, la superficie de la articulación de la patela, la tróclea femoral, el retináculo, las estructuras capsulares y la plica sinovial, y en segundo grado la fuerza del cuádriceps, el polo rotular superior, la bursa suprapatelar, la bursa prepatelar, el tendón patelar y bursa infrarotular profunda.

Para el diagnostico según Potter \& Sequeira 2002 (20), estudios de imágenes radiográficas generalmente no son necesarios para hacer el diagnóstico y recomendar tratamiento. Imágenes radiográficas deben ser consideradas para presentaciones no usuales y para individuos con el síndrome refractario al tratamiento conservador. Cohen et al. (21) afirman que, a pesar de innúmeros trabajos radiográficos para evaluación patelofemoral, generalmente ellos no ofrecen informaciones suficientes para indicar cirugía y meramente sugieren una anormalidad existente (20).

La radiografía es un complemento de la historia y el examen físico y debe realizarse en los pacientes con antecedentes de trauma o cirugía, derrame o que no mejoran con el tratamiento. La radiografía también puede ayudar a diagnosticar la rótula bipartita asintomática. En la mayoría de los pacientes con SCPF no son necesarias la tomografía computarizada ni las imágenes por resonancia magnética (RNM), aunque esta última puede ayudar a detectar las lesiones del cartílago articular, la condromalacia patelar, las fracturas de esfuerzo de la rótula y los cuerpos libres. También permite detectar el edema de la médula que compromete el aspecto medial patelar y el aspecto lateral del cóndilo femoral, y las lesiones del ligamento patelofemoral; estos hallazgos son sugestivos de subluxación o dislocación de la patela.

Como tratamiento para el dolor se utilizan analgésicos $\mathrm{y}$, aunque la indicación de AINEs para el SCPF es frecuente, hay poca evidencia que avale su eficacia. Para los pacientes con síntomas durante las actividades diarias y aquellos cuyos síntomas no desaparecen con la aplicación de hielo, se puede comenzar el tratamiento con AINEs o acetaminofeno. En este último caso se aplica AINEs durante tres semanas. El tratamiento tiene como objetivos, disminuir la sintomatología álgida-inflamatoria; reeducar las capacidades musculares; corregir la disfunción mecánica disminuyendo el "stress" patelofemoral.

Cuando la lesión compromete al cartílago en una condromalasia o pseudoartrosis, el glucosaminoglicano polisulfato (GAGPS) inhibe las enzimas proteolíticas, que degradan los proteoglicanos y el colágeno en el cartílago (22). También se ha demostrado que aumenta la tasa de síntesis y el grado de polimerización del ácido hialurónico en el líquido sinovial, lo que favorecería la reparación del cartílago. En los estudios en animales se ha demostrado que la aspirina inhibe los procesos enzimáticos destructivos en el cartílago (23).

Para controlar la inflamación se indica hielo 15 minutos post ejercicio o kinesiología, elevación y agentes antiinflamatorios no esteroides. Los ejercicios de movilidad articular deberían comenzar tempranamente para empezar la restauración de la función anatómica normal y el mantenimiento de la nutrición del cartílago articular. La restauración de la función del cuádriceps y la instauración de un programa de fortalecimiento muscular son fundamentales en la fase temprana realizando ejercicios de contracciones isométricas y ejercicios de cadena abierta de movimiento, siendo estas las más efectivas para fortalecer el cuádriceps, especialmente del vasto medial. En estos casos uno de los ejercicios más efectivos es la extensión de pierna en rotación lateral sin pesos. Otro elemento importante es la estimulación eléctrica que puede colaborar frente la inhibición refleja por dolor. 


\begin{tabular}{|c|c|c|}
\hline $\begin{array}{l}\text { Mal Alineamiento de la } \\
\text { Extremidad Inferior }\end{array}$ & $\begin{array}{l}\text { Lesión Asociada } \\
\text { (Over use) }\end{array}$ & Ajuste Sugerido \\
\hline $\begin{array}{l}\text { Alineamiento en valgo o } \\
\text { pie rotado hacia fuera. }\end{array}$ & $\begin{array}{l}\text { Dolor en la parte interna de la } \\
\text { rodilla. }\end{array}$ & $\begin{array}{l}\text { Ajustar los cleats o usar } \\
\text { órtesis en los zapatos. }\end{array}$ \\
\hline $\begin{array}{l}\text { Alineamiento en varo del } \\
\text { pie o "pie" rotado hacia } \\
\text { dentro. }\end{array}$ & $\begin{array}{l}\text { Dolor en la parte externa de } \\
\text { la rodilla y pierna. }\end{array}$ & $\begin{array}{l}\text { Ajustar los cleats o usar } \\
\text { órtesis en los zapatos. }\end{array}$ \\
\hline $\begin{array}{l}\text { Torsión tibial interna. } \\
\text { "Pierna rotada hacia } \\
\text { adentro". }\end{array}$ & $\begin{array}{l}\text { Dolor en la parte externa de } \\
\text { la rodilla y pierna. }\end{array}$ & $\begin{array}{l}\text { Ajuste en la alineación del } \\
\text { pedal y el zapato. }\end{array}$ \\
\hline $\begin{array}{l}\text { Torsión tibialexterna. } \\
\text { "Pierna rotada hacia } \\
\text { fuera". }\end{array}$ & $\begin{array}{l}\text { Dolor en la cara interna de la } \\
\text { rodilla. }\end{array}$ & $\begin{array}{l}\text { Ajuste en la alineación del } \\
\text { cleat del zapato. }\end{array}$ \\
\hline Dismetría en las piernas. & $\begin{array}{l}\text { Dolor en el talón de la pierna } \\
\text { más corta. }\end{array}$ & $\begin{array}{l}\text { Agregar una suela al zapato } \\
\text { para igualar la longitud de las } \\
\text { extremidades. }\end{array}$ \\
\hline $\begin{array}{l}\text { Malalineamiento de la } \\
\text { rótula. }\end{array}$ & Dolor de rodilla (rótula). & $\begin{array}{l}\text { Ajustes en la posición del } \\
\text { asiento y los zapatos con los } \\
\text { pedales. }\end{array}$ \\
\hline
\end{tabular}

Figura 2: Cuadro resumen de complicaciones, según mal alineamiento del miembro inferior.

Las consideraciones a tener en cuenta para evitar el dolor de la rodilla y de las dos lesiones de sobreuso mencionadas son, evitar el pedaleo que implique una flexión de rodilla mayor a $60^{\circ}$; realizar pedaleos en subidas y contra el viento a una cadencia alta, y utilizar los músculos posteriores del muslo y pierna para traccionar hacia arriba el pie, permitiendo liberar la tensión en tendones y músculos; realizar pedaleos sin movimientos laterales de la rodilla que alteren la mecánica del movimiento de la patela sobre el fémur, alinear la posición del pie de acuerdo a la línea recta natural del pedaleo.

\section{BIBLIOGRAFÍA}

1. Kyle, C.R. The aerodynamics of handlebars and helmets. Cycling Science. 1989. 1, 22-25.

2. Kyle, C.R. Wind tunnel tests of bicycle wheels and helmets. Cycling Science. 1990. 2, 27-30.

3. Jorge, M. \& Hull, M.L. Anakysis of EMG measurements during bicycle pedalling. Journal of biomechanics.1986. 19, 9, 683-694
4. McCormick, M. Seeking perfection with the force pedal. Velo-news. 1993. 8, 62-65.

5. Gonzalez, H. \& Hull, M.L. Multivariable optimization of cycling biomechanics. Journal of biomechanics.1989. 22, 11, 1151-1161.

6. Faria, I.E. \& Cavanagh, P.R. (1978). The Physiology and Biomechanics of Cycling. New York: John Wiley and Sons.

7. Cavanagh, P.R. \& Sanderson, D.J. (1986). I be biomechanics of cycling studies of the pedalling mechanics of elite pursuit riders. Ed: Science of cycling. Champaigh II. Human Kinetics. (27-30).

8. Murphy D.F., Connolly D.A.J., Beynnon B.D. Risk factors for lower extremity injury: a review of the literature br $\mathrm{j}$ sports med 2003;37:13-29.

9. Lancourt, JE, Cristini, JA Patella alta and patella infera. Their etiological role in patellar dislocation, chondromalacia, and apophysitis of the tibial tubercle J Bone Joint Surg Am 1975 57: 1112-1115. 
10. Khan KM, Bonar F, Desmond PM, Cook JL, Young DA,Visentini PJ, et al. Patellar tendinosis (jumper's knee): findings at histopathologic examination, US, and MR imaging. Victorian Institute of Sport Tendon Study Group. Radiology. 1996;200(3):821-7.

11. Blazina ME, Kerlan RK, Jobe FW, Carter VS, Carlson GJ. Jumper's knee. Orthop Clin North Am. 1973;4(3):665-78.

12. Pfeiffer R. Mangus B. Las lesiones deportivas Ed. Paidotribo. Barcelona 2000.

13. Buckwalter JA, Stanish WD, Rosier RN, Schenck RC Jr, Dennis DA, Coutts RD. The increasing need for nonoperative treatment of patients with osteoarthritis.

Clin Orthop. 2001 Apr;(385):36-45.

14. D’Amato M., Bach B.R.Jr. Lesiones de la rodilla. Brentz Brotzman S. Reabilitación Ortopédica Clínica. Ed. Elseiver. Madrid, 2005. Capítulo 4: 307-27.

15. Deveraux A.D., Lachmann S. Patellofemoral artharlgia in atletes attending a sport injury. clinic br. j sport med 1984; 18: 18-21.

16. Commandré, F.A., Viani, J.L., Zakarian, H, Denis, F. Patología de la rodilla del ciclista. Archivos de Medicina del Deporte Volumen $V$ - No 17 - Págs. 31-42.
17. Bentley G, Dow G: Current concepts of etiology and treatment of chrondramalacia patellae. Clin. Orthop. 1984. 189:209.

18. Starkey, Chad. Ryan, Jeff. (2001) Avaliações de Lesões Ortopédicas E Esportivas. São Paulo: Manole.

19. Wiberg G. Roentgenographic and anatomic studies on the femoropatellar joing. Acta Orthop Scand 1941; 12; 319-410.

20. Potter, Patrick J. Sequeira, Keith AJ. Patellofemoral Syndrome 2002. Disponible en: http://www.emedicine.com/articles.

21. Cohen, M; Abdalla, R J; Ferreti, M; Silva, P R. Síndrome Femoropatelar: Lesões nos Esportes-Diagnóstico-Prevenção-Tratamento. 2003. Rio de Janeiro: Revinter.

22. Kannus P, Natri A, Niittymäki S, Järvinen M. Effect of intraarticular glycosaminoglycan polysulfate treatment on patellofemoral pain syndrome - a prospective, randomized doubleblind trial comparing glycosaminoglycan polysulfate with placebo and quadriceps muscle exercises. Arthritis and Rheumatism 1992;35(9):1053-61.

23. Bentley G, Leslie IJ, Fischer D. Effect of aspirin treatment on chondromalacia patellae. Annals of Rheumatic Disease 1981;40(1):37-41. PMID: 7008711.

\footnotetext{
ABSTRACT

The cycling is certainly a sports activity of low impact, but carried out under inadequate conditions like a very heavy bicycle, using a low seat, misaligned the pedals, lack of a physical suitable condition or an incompatible morphology, is capable of provoking injuries for overcharge, that exist in wrists, arms, shoulders, column, knees and ankle. The most complicated is that of knee, the most common it are the Tendinopathy of the Patellar Tendon (TTP) and the Syndrome of the Patello-femoral Compression (SPFC). For both lesions in early stage conventional treatment works, but the important thing is prevention of the above mentioned injuries, having the suitable knowledge of the positions and loads of training adapted for every individual.
}

Key words: Cycling, Tendinopathy, Compretion patello-femoral.

\section{Dirigir correspondencia a:}

Fernando Rodríguez Rodríguez

Avenida el Bosque 1290, Santa Inés, Viña del Mar.

Escuela de Educación Física.

Pontificia Universidad Católica de Valparaíso

Chile.

fernando.rodriguez@ucv.cl 